\title{
INCOMPLETE CHROMOSOME PAIRING AND ITS RELATION TO CHIASMA LOCALISATION IN STETHOPHYMA GROSSUM SPERMATOCYTES
}

\author{
B. M. N. WALLACE and G. H. JONES \\ Department of Genetics, University of Birmingham, P.O.Box 363, Birmingham B15 $2 T T$ \\ Received 6.ix.77
}

\begin{abstract}
SUMMARY
Reconstructions of synaptonemal complexes (SC's) from serial sections of Stethophyma grossum spermatocytes demonstrate a clear correlation of chiasma distribution and the extent of pairing among different chromosome pairs. Proximal localisation of chiasmata in the eight longest chromosome pairs is associated with proximally localised incomplete development of the SC in eight bivalents. Up to three bivalents show complete SC development in EM reconstructions which parallels the non-localised chiasma formation shown by the three shortest bivalents at Metaphase I. The unpaired axial cores (AC's) of the incompletely paired bivalents were studied by a technique which revealed the nuclear membrane attachments of most AC's and allowed an assessment of the relative amounts of SC and AC. The extent of the correlation between chiasma localisation and incomplete pairing is examined and discussed.
\end{abstract}

\section{INTRODUCTION}

The Large Marsh Grasshopper, Stethophyma grossum, provides one of the classic examples of proximal chiasma localisation in the animal kingdom. Eight of the 11 telocentric autosomal bivalents formed in male meiosis almost invariably form single chiasmata which are localised very near to the terminal centromeres (White, 1936; Shaw, 1971; Perry and Jones, 1974). The underlying mechanism of chiasma localisation, both in this species and generally, is poorly understood though two broadly different mechanisms may be envisaged, one based on localised pairing of homologues, the other on localised exchange following complete pairing. These mechanisms may, however, be difficult to distinguish in practice because of the inherent difficulty of analysing pairing by light microscopy in many species, including Stethophyma grossum. Electron-microscopical (EM) analysis of the synaptonemal complex (SC) should facilitate this distinction in such cases, especially since the SC provides clear evidence of pairing, even in systems which are intractable by light microscopy.

A preliminary study of this problem in Stethophyma grossum males (Jones, 1973) concluded that localised pairing was unlikely to be the principal cause of chiasma localisation in this system. This conclusion was based on the observation of extensive SC formation in partially reconstructed pachytene nuclei and an apparent absence of unpaired axial cores (AC's). Indeed it was argued that pairing might be complete in all chromosome regions of Stethophyma grossum spermatocytes. A more thorough study of this problem has now been undertaken, based on reconstructions of several complete nuclei from serial EM sections; this considerably clarifies the relationship of chromosome pairing and chiasma localisation in this system. 


\section{Materials AND methods}

Testis follicles of a third instar male were fixed in 2.5 per cent glutaraldehyde in Sörensen's phosphate buffer $\left(p \mathrm{H} \mathrm{7)}\right.$ for 1 hour at $4^{\circ} \mathrm{C}$. Some of the follicles $\left(\right.$ Cell $\mathrm{D}^{\prime}$ ) were then post-fixed in 2 per cent osmium tetroxide in Sörensens phosphate buffer $(p \mathrm{H} 7)$ for 4 hours at $4^{\circ} \mathrm{C}$. Post-fixation was omitted in others (cells A, B, C) following the method of Sheridan and Barrnett (1969). The follicles were dehydrated in an alcohol series and left overnight in 1 per cent phosphotungstic acid (PTA) in absolute alcohol at $4^{\circ} \mathrm{C}$. They were then washed in absolute alcohol, and individual follicles embedded in Epon 812. Nuclei at appropriate stages of meiosis were identified from $2 \mu$ Epon-embedded survey sections stained with 4 per cent Giemsa in Sörensen's phosphate buffer $(p \mathrm{H} 8)$.

Serial sections of either $1000 \AA$ or $2500 \AA$ were cut on a Reichert Om U3 ultramicrotome, picked up on single slot copper grids and placed on a drop of water in a plastic petri dish before being transferred to filmed plastic rings as described by Wells (1974). The rings were covered with a film of Pioloform F (Wacker-Chemie, Munich). Grids were removed from the rings by inverting over a clean glass peg and viewed with a Phillips EM 300 electron microscope equipped with a goniometer stage, without further treatment.

Cells at different meiotic stages along the follicle were photographed on $70 \mathrm{~mm}$ film at 1800 . Prints were traced on to acetate sheets and each SC traced individually through the sections.

C-banding of mitotic chromosomes was performed by the standard $\mathrm{Ba}(\mathrm{OH})_{2}$-SSC-Giemsa technique.

\section{3, Results}

\section{(i) The karyotype in mitosis and meiosis}

The 23 chromosomes of Stethophyma grossum males comprise 11 pairs of autosomes and a single $\mathrm{X}$ chromosome. All chromosomes bear an apparently terminal centromere and the 11 autosome pairs show a very considerable size range (figs. 1 and 2, table 1). Giemsa C-banding of the karyotype reveals the existence of prominent procentric blocks of constitutive heterochromatin in all chromosomes. The sizes of these blocks show some variation from chromosome to chromosome and additionally some are subdivided into two or three bands of different sizes which characterise particular chromosomes. These procentric heterochromatic blocks presumably correspond to the densely staining blocks visible at diplotene of meiosis in this species (Shaw, 1971). The eight longest bivalents (L1-M8) each form a single proximally localised chiasma in male meiosis (fig. 2). The three shortest bivalents (M9, S10, S1l) do not conform to this pattern.

TABLE 1

Mean absolute lengths $(\mu)$ and relative lengths of the mitotic chromosomes of Stethophyma grossum based measurements of five $C$-banded and colchicine treated embryonic mitotic metaphases

\begin{tabular}{|c|c|c|c|c|c|c|c|c|c|c|c|}
\hline & L1 & L2 & L3 & M4 & M5 & M6 & M7 & M8 & M9 & $\mathrm{S} 10$ & S11 \\
\hline & $21 \cdot 4$ & $18 \cdot 7$ & $16 \cdot 2$ & $14 \cdot 5$ & $13 \cdot 2$ & $11 \cdot 4$ & $10 \cdot 2$ & $9 \cdot 3$ & $8 \cdot 9$ & $4 \cdot 7$ & $2 \cdot 3$ \\
\hline elative length & $9 \cdot 3$ & $8 \cdot 2$ & $7 \cdot 1$ & $6 \cdot 3$ & $5 \cdot 8$ & $5 \cdot 0$ & $4 \cdot 5$ & $4 \cdot 0$ & $3 \cdot 9$ & $2 \cdot 0$ & 1 \\
\hline
\end{tabular}




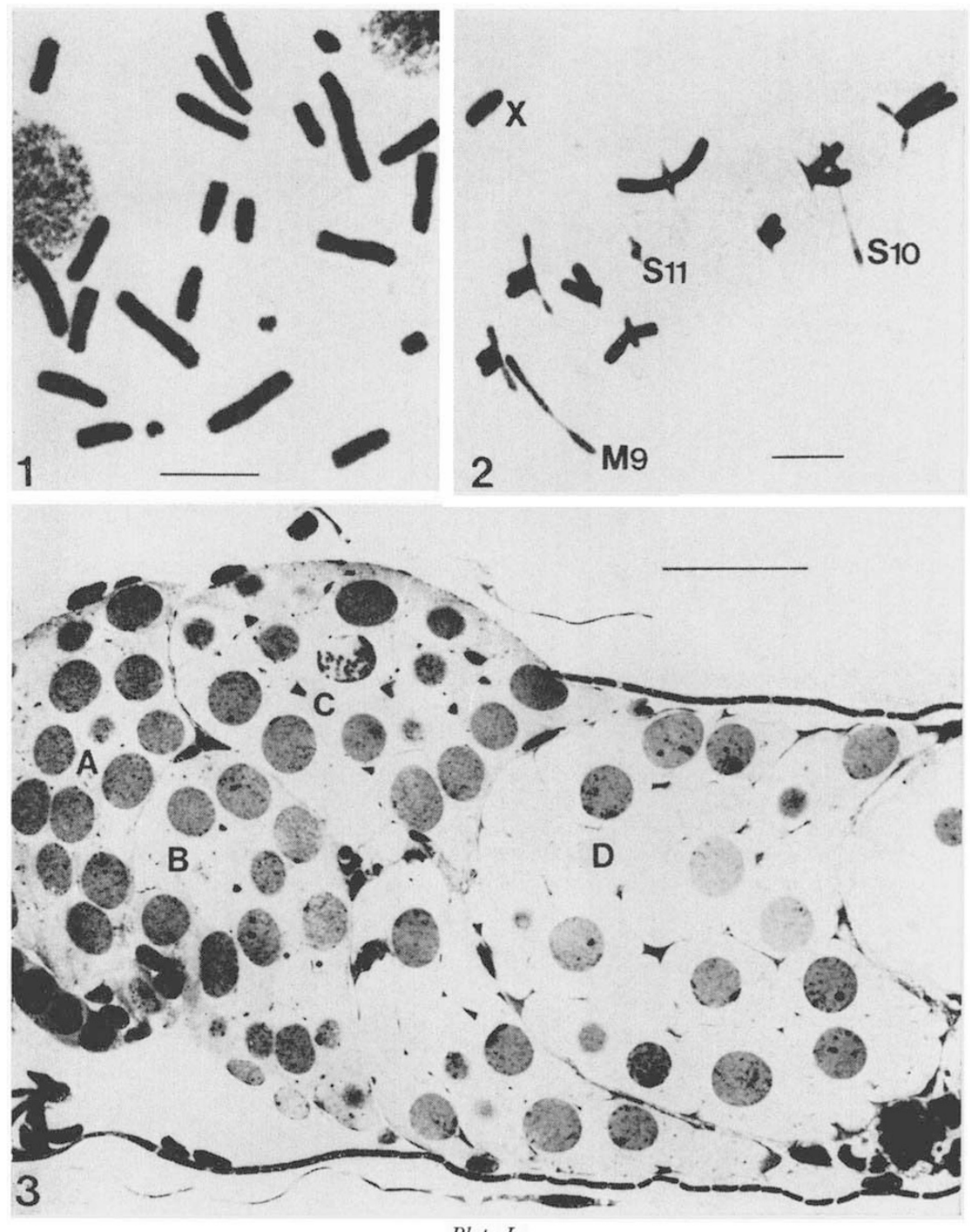

Plate I

Figs. 1 and 2.-The karyotype of Stethophyma grossum as seen in embryonic mitosis (fig. 1) and metaphase $\mathbf{I}$ of meiosis (fig. 2) (Bar $=20 \mu$ ).

Fig. 3.-Survey light microscope photograph of a $2 \mu$ Epon-embedded and giemsa-stained section of the testis follicle which provided most of the material for this study. Cysts $A$, $\mathrm{B}$ and $\mathrm{C}$ yielded the reconstructed nuclei $\mathrm{A}, \mathrm{B}$ and $\mathrm{C}$ and cyst $\mathrm{D}$ is at an equivalent stage to nucleus $\mathrm{D}^{\prime}$ which came from a different follicle $(\mathrm{Bar}=50 \mu)$. 


\section{Plate II}

FIG. 4.--Electron micrograph of nucleus A (glutaraldehyde fixed without osmium tetroxide, PTA stained, section thickness $\bumpeq 2500 \AA$ ), showing stretches of SC and AC, two nucleoli (N) and a single knobbed SC attachment (KSCa) (Bar $=3 \mu)$.

FIGs. 5-7.-Details of the various types of SC and AC attachments to the nuclear membrane in $2500 \AA$ sections of glutaraldehyde fixed and PTA stained material. $\mathrm{KSCa}=$ knobbed SC attachment; $\mathrm{PSCa}=$ plain SC attachment; $\mathrm{ACa}=\mathrm{AC}$ attachment. Note the complex knob structure evident in fig. 7. The apparent separation of the other $\mathrm{SC}$ attachment from the nuclear membrane in this figure is due to the thickness of the section $(\operatorname{Bar}=2 \mu)$. 

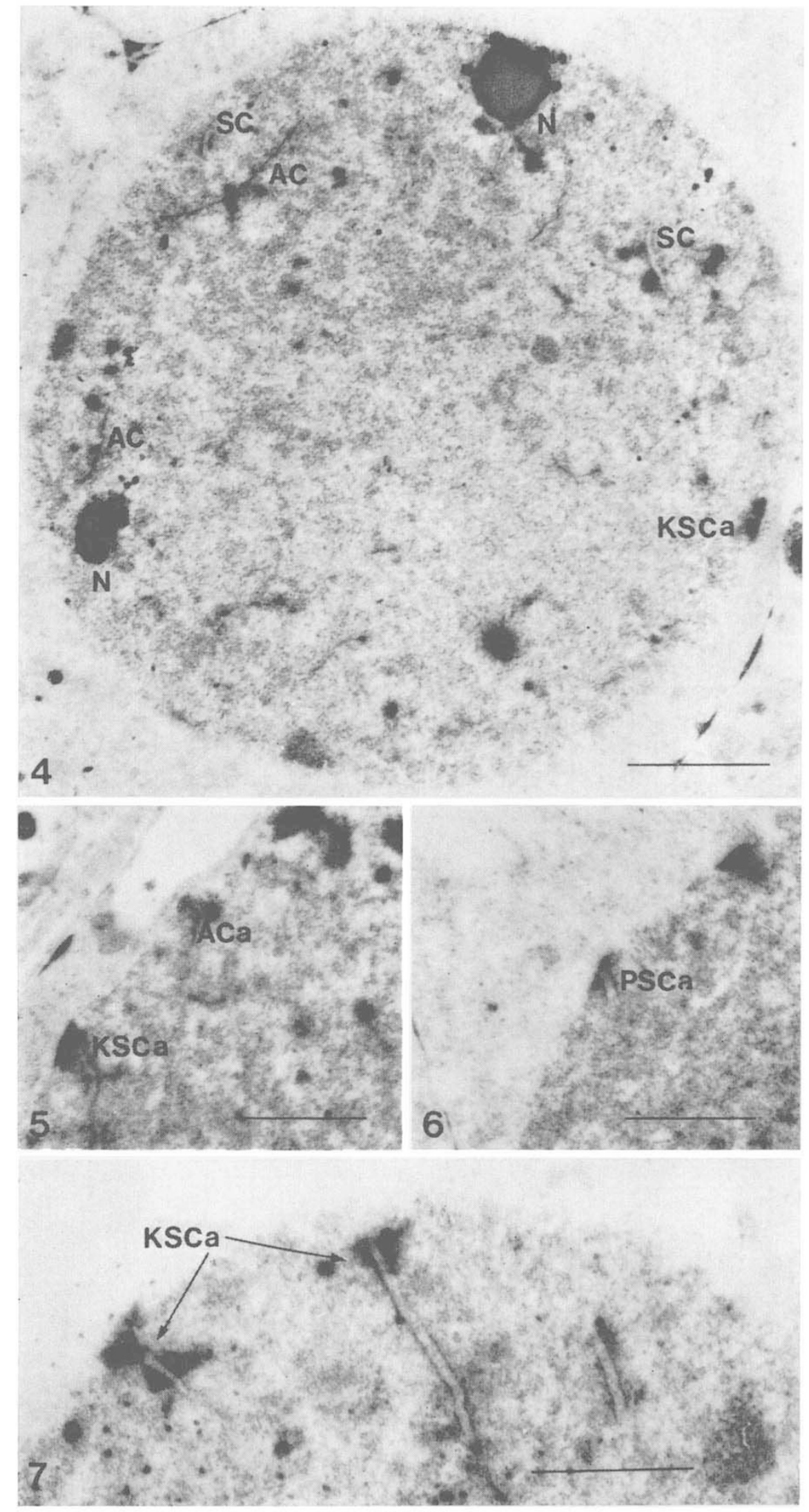
The M9 usually forms a single distal chiasma but quite commonly also forms two chiasmata, when they generally occur distally and interstitially or distally and proximally. The two smallest bivalents (S10, S1l) show very little tendency to chiasma localisation and almost invariably form a single chiasma which may form proximally, interstitially or distally (Perry and Jones, 1974).

\section{(ii) The meiotic sequence}

The developmental sequence of male germ line cells in Stethophyma grossum has been described in earlier papers (Janssens, 1924; McGlung, 1927) and will be described only briefly here. The distal blind end of each testis follicle contains groups (cysts) of spermatogonia which show synchronous development and undergo periodic increases in cell number by mitotic division. Spermatogonial stages are characterised by a pronounced condensation of the chromatin (prochromosomes) which persists into the immediately pre-meiotic spermatocytes. This is followed by an unravelling stage which leads to a typical leptotene appearance of distinctive fine threads with prominent chromomeres. Between leptotene and diplotene no stages representing the zytogene and pachytene of textbook accounts of meiosis can be seen. Instead the chromosomes become progressively diffuse and the nucleus enlarges at the same time. This diffuse stage, which was long ago described by Wilson (1925) — see also John and Lewis (1965) and Klasterska (1976) - occupies the greater part of prophase I and at its greatest development very little evidence of chromosome organisation is detectable by light microscopy. Eventually the chromosomes condense and reappear to give a typical diplotene appearance.

Fig. 3 shows a low magnification survey photograph of the testis follicle which provided most of the material for this study. Cysts A, B and C were the sources of reconstructed nuclei $\mathrm{A}, \mathrm{B}$ and $\mathrm{C}$. Cyst $\mathrm{A}$ contains nuclei at an early post-leptotene diffuse stage; they contain a fine network of chromosome threads and chromomeres but these features are much less distinct than in leptotene. Nuclei in cysts B and C are at a more advanced stage of diffuseness but still retain some traces of fibrillar chromosome organisation, while in cyst D the diffuse stage is fully developed, cells and nuclei are considerably enlarged and there is little evidence of threads or chromomeres. The reconstructed nucleus $D^{\prime}$ was from a different follicle but from a cyst at roughly the same stage as cyst $\mathrm{D}$ in fig. 3 .

\section{(iii) Observations on chromosome pairing}

EM observations reveal the presence of substantial amounts of both synaptonemal complex (SC) and densely staining single cores (axial cores = $\mathrm{AC}$ ) in all four nuclei studied (e.g. fig. 4). AC's are particularly prominent in nuclei $\mathrm{A}, \mathrm{B}$ and $\mathrm{C}$, probably as a result of the combination of fixation and staining employed, together with an extra-thick section thickness $(\bumpeq 2500 \AA)$. The AC's resemble those normally seen in leptotene or very early zytogene stages (e.g. Moens, 1969; Church, 1976) or in asynaptic mutants which are genetically defective in pairing (La Cour and Wells, 1970), except that they are particularly fine and attenuated and lack the distinctive chromatin sheath normally associated with AC's, these features

$40 / 3-D$ 
presumably reflecting the extreme development of the diffuse stage in this system. The mixture of SC's and AC's therefore gives a clear indication that pairing is incomplete in the nuclei studied although they represent much later stages than those normally associated with the presence of AC's. In some cases $A C$ 's could be seen to be continuous with the free ends of SG's, but it was impossible to trace AC's very far because of their extremely tenuous nature and the particular problem of following them when sectioned transversely. However, AG attachments to the nuclear membrane were located and the AC's were reconstructed back from them as far as possible. SC's are more distinctive structures than $A C$ 's and hence are more readily traced through serial sections; consequently every effort was made to reconstruct completely the SG complement of each nucleus.

The reconstructed SC's and AC attachments from nuclei A-D' are summarised in figs. 8-10 and table 2. Surveying the data as a whole, 11 or 12 separate stretches of SC appear in each reconstructed nucleus, while the number of recorded SC attachments to the nuclear membrane is either 13 or 14 per nucleus. The simplest interpretation of these reconstructions is that three bivalents are completely paired in each nucleus, each of these

TABLE 2

A summary of the reconstructions of SC and AC structures from serial EM sections of four spermatocyte nuclei

\begin{tabular}{|c|c|c|c|c|c|c|c|c|c|}
\hline \multirow{2}{*}{ Nucleus } & \multirow[b]{2}{*}{$\begin{array}{l}\text { Section no. } \\
+ \text { thickness }\end{array}$} & \multicolumn{2}{|c|}{ SC attachments } & \multicolumn{3}{|c|}{ SC's (no. and length $[\mu]$ ) } & \multirow[b]{2}{*}{$\begin{array}{c}\mathrm{AC} \\
\text { attachments }\end{array}$} & \multicolumn{2}{|r|}{ Nucleoli } \\
\hline & & knobbed & plain & 2-attach's & & -attach & & No. & $\begin{array}{l}\text { Distance to knob } \\
\text { Chrom. } 1(\mu)\end{array}$ \\
\hline A & $72(2500 \AA)$ & 11 & 3 & $3\left\{\begin{array}{l}17 \cdot 8 \\
19 \cdot 8 \\
27.5\end{array}\right.$ & 8) & & 14 & 4 & $10 \cdot 2$ \\
\hline B & $73(2500 \AA)$ & 11 & 3* & $3\left\{\begin{array}{l}14 \cdot 3 \\
17 \cdot 2 \\
34 \cdot 9 \\
17 \cdot 3\end{array}\right.$ & 8 & $\stackrel{\bumpeq}{\bumpeq}$ & 13 & 4 & $8 \cdot 1$ \\
\hline C & $63(2500 \AA)$ & 10 & 3 & $2\left\{\begin{array}{l}29 \cdot 1 \\
18 \cdot 1\end{array}\right.$ & $9 \dagger$ & & 16 & 4 & $8 \cdot 7$ \\
\hline$D^{\prime}$ & $172(1000 \AA)$ & 11 & 3 & $2\} 22.9$ & 10 & & 4 & 4 & $10 \cdot 1$ \\
\hline
\end{tabular}

* One plain end (11P, fig. 9a) surmised, due to missing section.

$\dagger$ Two SC's represented by attachments only; this nucleus also contains a long SG stretch with no apparent attachment.

having two attachments to the nuclear membrane. The remaining eight bivalents of each nucleus are incompletely paired and have SC attachments to the nuclear membrane at one end only. The exceptions (nuclei $\mathrm{G}$ and $\mathrm{D}^{\prime}$ ) diverge only slightly from this pattern and are accountable in terms of a missing $\mathrm{SC}$ attachment (nucleus $\mathrm{C}$ ) or missing $\mathrm{SC}$ connecting two attachments (nuclei $\mathrm{C}$ and $\mathrm{D}^{\prime}$ ) both of which could result from occasional poor development of the SC or from technical factors such as poor preservation of SG or failure to reconstruct accurately. Occasional short stretches of $\mathrm{SC}$ are encountered which are not attached to the nuclear membrane and have no obvious connection with other SC's. These are interpreted as sporadic regions of pairing, remote from the main paired regions.

The completely paired bivalents show a clear distinction between their 
two ends, each bivalent having one knobbed and one plain attachment (see figs. 5-7). Since each of the incompletely paired bivalents has a prominent knobbed attachment it is probable that knobbed attachments correspond to centromeric ends and the knobs themselves correspond to procentric heterochromatin. Indeed, some knobs show a distinctly compound structure (e.g. fig. 7) which parallels the subdivision of the procentric mitotic C-bands. Plain ends evidently correspond to telomeres but it is interesting to note that even these have a distinctive structure involving a much reduced plaque-like knob where each lateral element of the SC joins the membrane (fig. 6).

The lengths of the SC's were measured for each of the two or three completely paired bivalents in each nucleus (table 2). There are reasonable grounds for identifying these SC's with the M9, S10 and S1l bivalents since these are the only bivalents which regularly form distal and interstitial chiasmata as well as proximal chiasmata. Bivalent identification among this group can probably be taken a stage further since they show a gradient of SC lengths ranging from $14 \mu$ to $34 \mu$ of which the S11 presumably corresponds to the shorter length and the M9 to the longer. For the incompletely paired bivalents, the range of SC lengths was estimated rather subjectively (table 2) since there was no way of identifying particular bivalents among this group with the exception of one nucleolus organising bivalent. These estimates are sufficiently accurate to assert that no largescale systematic variation in SC length can be detected between the different nuclei studied, despite the variation in cytological stage. This suggests that pairing has reached its maximum already in the earliest stage studied (nucleus A) and that no further extension of pairing is likely to occur in stages later than nucleus $\mathrm{D}^{\prime}$. Such a conclusion is also supported by many separate observations (but not reconstructions) of restricted SC development in other spermatocytes in a range of developmental stages from a number of different males. The duration of pachytene in species showing complete pairing is at least as long as and frequently exceeds the duration of zygotene (e.g. Franchi and Mandl, 1962; Moens, 1968) and therefore the chances of overlooking a completely paired pachytene stage in Stethophyma are very small. Furthermore, an examination of the earliest resolvable diplotene stages in Stethophyma grossum males shows that homologues are widely separated from their earliest appearance (see fig. 7, Jones, 1973) which is again indicative of incomplete pairing.

Systematic reconstruction of AC's was restricted to enumeration of $\mathrm{AC}$ attachments to the nuclear membrane (figs. 8-10b). Each AC ends in a small but distinct plaque on the nuclear membrane (fig. 5) which is clearly analogous in structure to the plain SC attachments, thus confirming that the $\mathrm{AC}$ attachments correspond to the unpaired telomeres of the incompletely paired L1-M8 bivalents. $\mathrm{Up}$ to $16 \mathrm{AG}$ attachments were located which is the maximum number expected if each of the incompletely paired bivalents has two $\mathrm{AC}$ ends attaching to the nuclear membrane. Fewer than 16 were found in other nuclei (see table 2) but this is hardly surprising in view of their tenuous nature. The very low number (4) observed in nucleus $D^{\prime}$ could be a reflection of the late stage of this nucleus or the different technique adopted. In general, the numbers of AC attachments observed is fully consistent with the eight longer bivalents of the complement being incompletely paired but nevertheless attaching by both 

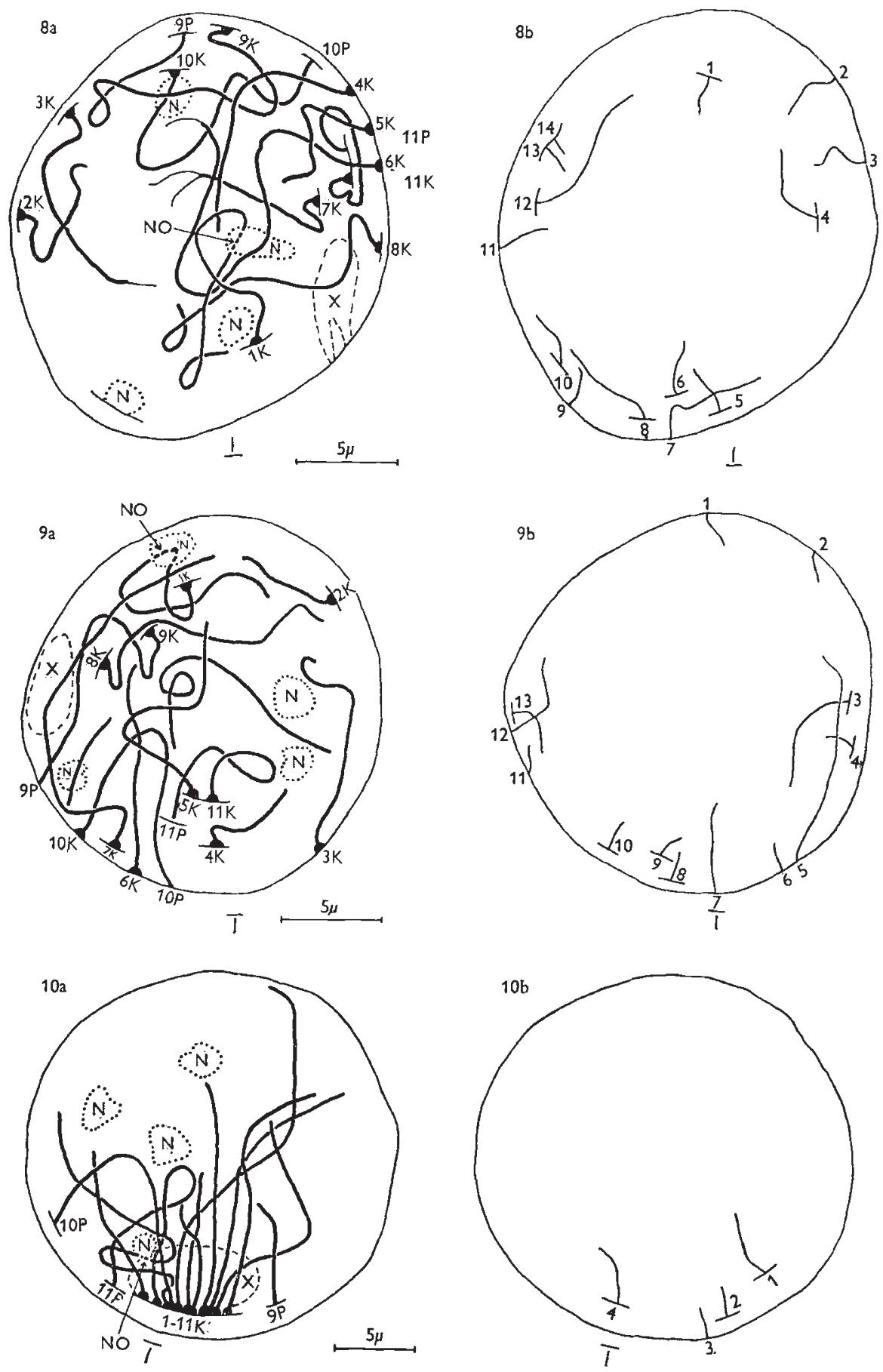

Figs. 8-10.-Reconstructions of SC's (figs. 8-10a) and AG attachments (figs. 8-10b) from nuclei A (fig. 8ab), B (fig. 9ab) and D' (fig. 10ab). SC's are represented for the sake of clarity as single thick lines and AC's as single thin lines. Knobbed (K) and plain (P) $\mathrm{SC}$ attachments are identified and also numbered, but on an arbitrary basis except that the completely paired bivalents are as far as possible allotted numbers 9,10 and 11 and the identifiable nucleolus organising chromosome is allotted number 1. AC attachments are arbitrarily numbered. $\mathrm{N}=$ nucleolus, $\mathrm{NO}=$ nucleolus organiser, $\mathrm{X}=\mathrm{X}$ chromatin. 
ends of each chromosome to the nuclear membrane. AC's were reconstructed back from their attachments as far as possible (figs. 8-10b) but in most cases they could only be traced a short distance (about 2-5 $\mu$, exceptionally $12 \mu$ ) before they turned perpendicular to the plane of sectioning and were lost.

The extent of pairing in the eight incompletely paired bivalents cannot be measured directly because of the impracticality of reconstructing chromosome pairs completely from end to end through SC and AC. As noted earlier, this is a particular difficulty of this system and results from the unusual attenuation of the ACs and the lack of an investing chromatin sheath, both of which are thought to reflect the extreme development of the diffuse stage in these spermatocytes. An indirect approach to this problem was followed by assessing the relative proportion of SC to AC over these eight pairs of chromosomes using a sampling technique. Since these AC's are very difficult to detect when sectioned transversely, observations on AC's and SC's were restricted to stretches sectioned longitudinally or obliquely and for this purpose a minimum length of $0.4 \mu$ of AC or SC was set. Any stretches of $\mathrm{AC}$ or $\mathrm{SC}$ equalling or exceeding this length in photomicrographs of serial EM sections were recorded and measured, disregarding any SG stretches previously identified as belonging to the $\mathrm{M} 9, \mathrm{~S} 10, \mathrm{~S} 11$ group. This approach assumes that SC is not more or less likely to be transversely or longitudinally sectioned than is AC. This assumption is not easily tested since it depends on a number of complex variables such as the relative dispositions of $\mathrm{SC}$ and $\mathrm{AC}$ within the nucleus and the plane of sectioning relative to these. Nevertheless, it is felt that in general terms this assumption is valid since $\mathrm{SC}$ and $\mathrm{AC}$ do not have strikingly different distributions or orientations within the nuclei studied and furthermore any possible effect of the plane of sectioning will be reduced because it varies between the different nuclei studied.

The aggregate lengths of $\mathrm{SC}$ and $\mathrm{AC}$ measured in complete section series of nuclei, A, B and $\mathrm{C}$ are given in table 3. Taking nucleus $\mathrm{B}$ as an example, the ratio of measured $\mathrm{AC}$ to $\mathrm{SC}$ is $3.27: 1$ but since $\mathrm{AC}$ material is unpaired and contributes twice, the corrected ratio of unpaired to paired chromosome regions averaged over the eight longer bivalents of this nucleus is $1.63: 1$. Nuclei A and $\mathrm{C}$ yielded similar values for the relative proportions of $\mathrm{SC}$ and $\mathrm{AC}$ and the values for all three nuclei together suggest that the incompletely paired $\mathrm{L}$ and $\mathrm{M}$ bivalents are paired on average for about 40 per cent of their length. A possible criticism of this conclusion is that since these chromosomes have not been reconstructed entirely, it assumes

TABLE 3

Relative amounts of SC and $A C$ observed in spermatocyte nuclei

\begin{tabular}{|c|c|c|c|c|c|c|}
\hline & \multicolumn{2}{|c|}{$\mathrm{SC}\left(\mathrm{L}_{1}-\mathrm{M} 8\right)$} & \multicolumn{2}{|r|}{$\mathrm{AC}$} & & \\
\hline Nucleus & $\begin{array}{l}\text { Aggregate } \\
\text { length }(\mu)\end{array}$ & $\begin{array}{c}\text { No. observed } \\
(>0 \cdot 4 \mu)+\text { mean } \\
\text { length }(\mu)\end{array}$ & $\begin{array}{l}\text { Aggregate } \\
\text { length }(\mu)\end{array}$ & $\begin{array}{c}\text { No. observed } \\
(>0 \cdot 4 \mu)+\text { mean } \\
\text { length }(\mu)\end{array}$ & $\begin{array}{l}\text { Ratio, } \\
\text { SC : AC }\end{array}$ & $\begin{array}{l}\text { Inferred } \\
\% \text { pairing }\end{array}$ \\
\hline $\begin{array}{l}\text { A } \\
\text { B } \\
\text { C }\end{array}$ & $\begin{array}{l}59 \cdot 0 \\
74 \cdot 8 \\
64 \cdot 5\end{array}$ & $\begin{array}{l}77(0.77) \\
96(0.78) \\
73(0.88)\end{array}$ & $\begin{array}{l}185 \cdot 7 \\
245 \cdot 2 \\
139 \cdot 2\end{array}$ & $\begin{array}{l}214(0.87) \\
278(0.88) \\
146(0.95)\end{array}$ & $\begin{array}{l}3 \cdot 15: 1 \\
3 \cdot 27: 1 \\
2 \cdot 15: 1\end{array}$ & $\begin{array}{l}38 \cdot 83 \\
37 \cdot 95 \\
48 \cdot 19\end{array}$ \\
\hline
\end{tabular}


that $\mathrm{SC}$ or $\mathrm{AC}$ develops in all chromosome regions. If some unpaired regions failed to develop AC's, the degree of pairing would be overestimated by this method, but it would be necessary to study entire bivalents in whole mounts of spread bivalents to exclude this possibility conclusively.

The arrangement of $\mathrm{SC}$ and $\mathrm{AC}$ attachments was also recorded in each reconstructed nucleus. SC attachments show a distinctly ordered and non-random arrangement, but their arrangement also varies depending upon the stage. In the earliest stage studied (nucleus $\mathrm{A}$ ) the $\mathrm{SC}$ attachments are widely scattered in the hemisphere farthest from the centrioles and the adjoining mitochondrial mass. Nuclei $B$ and $C$ which are known to be more advanced from their positions in the follicle show a progressive rearrangement of the $\mathrm{SC}$ attachments towards the centriolar hemisphere, with nucleus $\mathrm{C}$ which is probably the more advanced of these two showing a more distinct clustering. The latest stage studied $\left(D^{\prime}\right)$ shows a very pronounced ordering of the SC attachments; all 11 knobbed attachments are clustered in a very small zone immediately adjacent to the centrioles while the three plain SC attachments are segregated from this group and from one another. This arrangement of knobbed and plain SC ends closely resembles that observed in Brachystola magna spermatocytes (Church, 1976).

The arrangement of $\mathrm{AC}$ attachments also shows a progressive change with advancing stage. Nucleus A shows the least degree of order, with $\mathrm{AC}$ attachments widely and apparently randomly scattered around the nuclear membrane. Both nuclei B and $\mathrm{C}$ show some degree of order with $\mathrm{AC}$ attachments predominantly in the centriolar hemispheres but in neither case forming a close association with the $\mathrm{SC}$ attachments. In nucleus $\mathrm{D}^{\prime}$ only four AC attachments were seen but these were grouped at the centriolar pole, though not as compactly as the SC attachments.

Some impression of the distribution of SC and AC through the nuclei can be obtained by plotting the occurrence of $\mathrm{SC}$ and $\mathrm{AC}$ against position in the nucleus, represented by advancing section number (fig. 11). For example, in nucleus $\mathrm{C}, \mathrm{SC}$ and $\mathrm{AC}$ show different but to some extent complementary distribution curves. $\mathrm{SC}$ is relatively abundant in the centriolar region (sections 45-60) while AC's are scarce or absent here. Further out, AC's and SC's occur together while at the opposite end of the nucleus from the centrioles, AC's occur more frequently than SC's. Bearing in mind the relative positions of $\mathrm{SC}$ and $\mathrm{AC}$ attachments in this nucleus, this suggests that SC's extend variable distances from their knobbed attachments towards the other extremity of the nucleus, eventually transforming into axial cores which extend back towards the centriolar region and terminate at attachment sites in the centriolar hemisphere.

Figs. 8-10a show the positions of the $\mathrm{X}$ chromosomes and nucleoli within the nuclei studied. The $\mathrm{X}$ chromosome appears as a relatively unstructured and dispersed area of condensed chromatin about $7 \mu$ in diameter. Its position in the nucleus varies with cytological stage; in nuclei $A, B$ and $C$ its position is variable but in nucleus $D^{\prime}$ it is found in a juxta-centriolar position immediately adjacent to the cluster of knobbed $\mathrm{SC}$ attachments. Four nucleoli are found regularly in each nucleus but only one of these is regularly associated with SC. This appears to be a distinct nucleolus organising (NO) region since it occurs at a reasonably constant distance from a knobbed SC attachment (table 2). This is therefore the only incompletely paired bivalent which can be consistently recognised. 


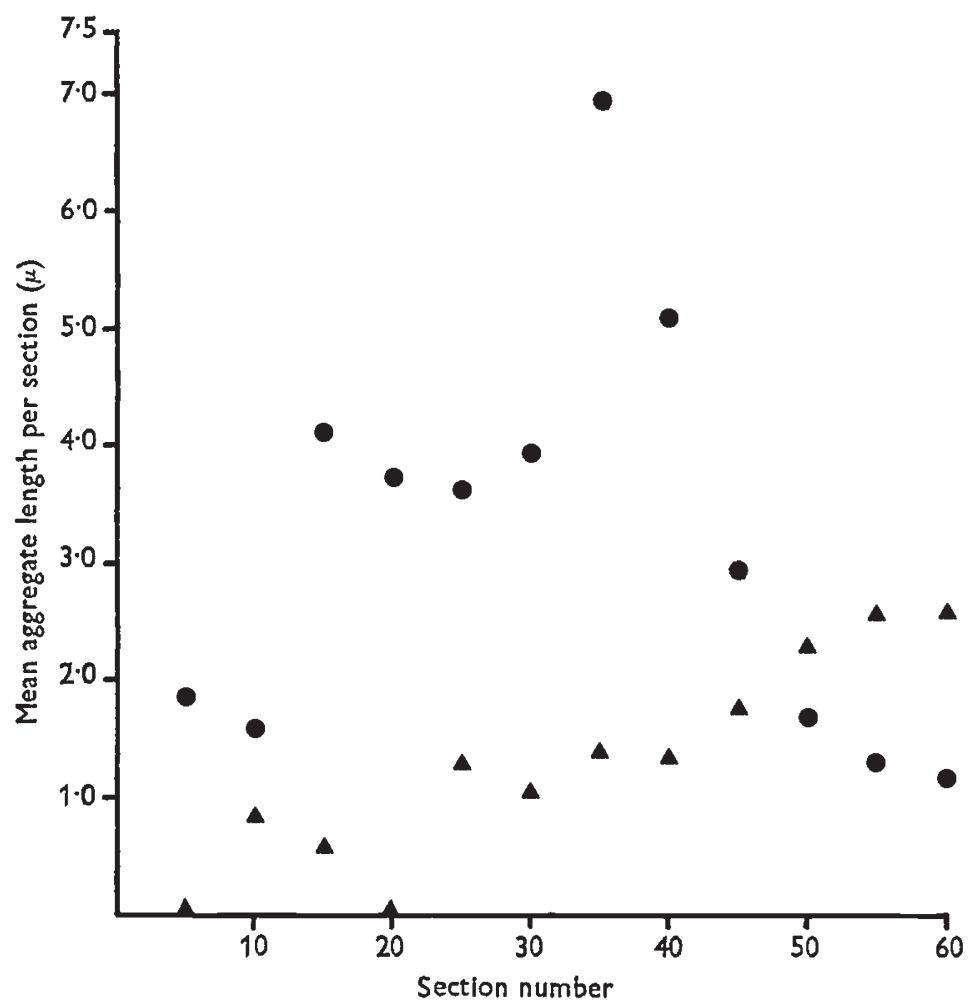

Frg. 11.-Aggregate $S C$ and $A C$ lengths plotted against advancing section number from a serial EM reconstruction of nucleus C. Each point represents the mean aggregate length of SC and AC from five consecutive sections, in order to minimise fluctuations due to sampling variation ( $=$ AC's; $\Delta=$ SC's).

\section{Discussion}

The clearest conclusion to emerge from this study is that a correlation exists between pairing behaviour and the localisation of chiasmata in Stethophyma grossum spermatocytes. In EM reconstructions, three bivalents are completely paired and eight bivalents are paired only at their proximal ends, while at Metaphase I three bivalents show relatively unlocalised chiasma formation and eight bivalents each form a single proximally localised chiasma. The identity of the three completely paired SG's in reconstructions with the three shortest bivalents at Metaphase I is conjectural, but the coincidence of pairing behaviour and chiasma behaviour cannot be readily explained otherwise. Essentially similar findings to those described here have been independently obtained by Fletcher (1978) working at the University of East Anglia. However, despite the similarity of our aims, the two studies show some interesting points of difference in methods, results and interpretation and for maximum information both papers should be consulted.

The situation in Stethophyma grossum males is clearly analogous to those previously described by light microscopy in Fritillaria species (Darlington, 
1935) and in the grasshopper Chloealtis conspersa (John, 1976). It is a remarkable fact, however, that incomplete pairing was also recognised and described in Stethophyma grossum males more than 50 years ago. In his classic analysis of meiosis in grasshopper species Janssens (1924) states (in translation) "In Stethophyma grossum pairing is only produced at certain places and otherwise chromosomes remain independent even though they may sometimes lie parallel", a conclusion later supported by Darlington (1931).

We may next inquire if incomplete pairing is a sufficient explanation of the proximal chiasma localisation which characterises this system. Two approaches to this question have been followed, yielding somewhat conflicting estimates of the extent of pairing. The ratios of SC to AC obtained by the sampling method described earlier suggest that the incompletely paired bivalents (L1-M8) are paired for about 40 per cent of their lengths, on average. This estimate, however, depends on the untested assumption that all unpaired regions develop AC's. On the other hand, it has been observed that the incompletely paired bivalents each form about as much SC (approximately 10-20 $\mu$ ) as the S1l bivalent and this suggests that only a region comparable in length to the $\mathrm{Sll}$ chromosome is actually paired in each of these much longer bivalents. Clearly this gives a very different estimate of the extent of pairing than the average figure of 40 per cent quoted above. To be precise, since the mitotic length of chromosome S1l is about 16 per cent of the mean mitotic length of the eight incompletely paired chromosomes this implies that this group of chromosomes is about 16 per cent paired on average (but with a range from 25 per cent (M8) to 11 per cent $(\mathrm{Ll}))$. However, these estimates assume that $\mathrm{SC}$ length is directly proportional to mitotic chromosome length and while this has been clearly demonstrated in a number of species (Moses and Counce, 1976) it is possible that it does not apply universally. In this particular system it is difficult to confirm directly because so many of the bivalents are incompletely paired. The completely paired bivalents (S1 1, S10, M9) give an ambiguous result since the ratio of their $\mathrm{SC}$ lengths (1:1.2:1.8, nuclei $\mathrm{A}$ and $\mathrm{B}$, table 2) is rather different from the corresponding ratio of mitotic lengths ( $1: 2 \cdot 0: 3 \cdot 9$, table 1 ), but these ratios are nevertheless sufficiently alike for the differences to be possibly explained by errors of measurement or differential contraction of the S11, S10 and M9 chromosomes during mitosis.

The degree of chiasma localisation in bivalents Ll-M8 is difficult to quantify because of differential stretching (at metaphase I) and differential condensation (at diplotene) of chromatin on either side of the chiasmata; it is however, quite variable. In some bivalents the chiasma forms very close to the centromere and only finely attenuated threads connect the centromere to the rest of the bivalent at metaphase I. In other bivalents the chiasma forms some way from the centromere, but in no case does the centromere to chiasma distance exceed the length of the Sll chromosome, although it frequently approaches or equals this distance (see fig. 2 and figs. 3 and 4, Perry and Jones, 1974). There is therefore a fair degree of correspondence between the average extent of pairing in the Ll-M8 bivalents (i.e. 16 per cent), assuming that SC lengths are proportional to the length of paired chromosomes, and the degree of chiasma localisation at metaphase I. However, direct observations on relative amounts of SC and AC in three nuclei suggest that pairing in these bivalents $(\bumpeq 40$ per cent) may 
considerably exceed the localisation of chiasmata. Further work, preferably based on whole mount spreads of meiotic bivalents should resolve this uncertainty.

An association between incomplete pairing and localised chiasmata is capable of interpretation in a number of ways. One point of view is that since pairing of homologues is clearly a pre-condition for chiasma formation, incomplete pairing could determine chiasma localisation (e.g. Henderson, 1969). Alternatively, chiasma localisation could depend on localisation of exchange events and incomplete pairing could have evolved secondarily (e.g. John, 1976). It is difficult to decide between these hypotheses, but the observation that elevated temperatures applied to spermatocytes in pre-meiosis can induce additional pairing and chiasma formation in distal and interstitial regions of $L$ and $M$ bivalents (Perry, 1974) suggests that incomplete pairing conditions chiasma distribution in this system and not vice-versa.

Acknowledgments.-We are grateful to the Science Research Council for financial support.

\section{REFERENGES}

$\mathrm{CHURCH}_{3}$ K. 1976. Arrangement of chromosome ends and axial core formation during early meiotic prophase in the male grasshopper Brachystola magna by 3D, E.M. reconstruction. Chromosoma (Berl.), 58, 365-376.

DARLINGton, c. D. 1931. Meiosis. Biol. Rev., 6, 221-264.

darlington, c. D. 1935. The internal mechanics of the chromosomes. II. Prophase pairing at meiosis in Fritillaria. Proc. Roy. Soc. (Lond.), B., 118, 59-73.

FLETCHER, H. L. 1978. Localised chiasmata due to partial pairing: a 3D reconstruction of synaptonemal complexes in male Stethophyma grossum. Chromosoma, 65, 247-269.

FRANCHI, L. L., AND MANDL, A. M. 1962. The ultrastructure of oogonia and oocytes in the foetal and neonatal rat. Proc. Roy. Soc. (Lond.), B, 157, 99-114.

henderson, s. A. 1969. Chiasma localisation and incomplete pairing. Chromosomes Today, 2, 56-60 (C. D. Darlington and K. R. Lewis, eds.). Edinburgh, Oliver \& Boyd.

JANsSENS, F. A. 1924. La chismatypie dans les insectes. La Cellule, 34, 135-359.

JoHN, B. 1976. Myths and mechanisms of meiosis. Chromosoma (Berl.), 54, 295-325.

JOHN, B., AND LEWIS, K. R. 1965. The Meiotic System. Protoplasmatologia VI, F1. Wien, Springer-Verlag.

JONEs, G. H. 1973. Light and electron microscope studies of chromosome pairing in relation to chiasma localisation in Stethophyma grossum (Orthoptera: Acrididae). Chromosoma (Berl.), 42, 145-162.

KLASTERSKA, I. 1976. A new look at the role of the diffuse stage in problems of plant and animal meiosis. Hereditas (Lund), 82, 193-204.

LA COUR, L. F., AND WELLs, B. 1970. Meiotic prophase in anthers of asynaptic wheat. Chromosoma (Berl.), 29, 419-427.

MCCLUNG, c. E. 1927. Synapsis and related phenomena in Mecostethus and Leptysma (Orthoptera). 7. Morph., 43, 181-265.

MoENs, P. B. 1968. The structure and function of the synaptinemal complex in Lilium longiflorum sporocytes. Chromosoma (Berl.), 23, 418-451.

MOENS, Р. в. 1969. The fine structure of meiotic chromosome polarisation and pairing in Locusta migratoria spermatocytes. Chromosoma (Berl.), 28, 1-25.

MOSES, M. J., AND COUNCE, s. J. 1976. Analysis of synaptonemal complex karyotypes in five mammals. 7. Cell. Biol., 70, 131a.

PERry, P. E. 1974. Ph.D. Thesis. University of Birmingham.

PERRY, P. E., AND JONES, G. H. 1974. Male and female meiosis in grasshoppers. I. Stethophyma grossum. Chromosoma (Berl.), 47, 227-236.

SHAW, D. D. 1971. The supernumerary segment system of Stethophyma. II. Heterochromatin polymorphism and chiasma variation. Chromosoma (Berl.), 34, 19-39.

SHERIDAN, W. F., AND BARRNETT, R. J. 1969. Cytochemical studies on chromosome ultrastructure. J. Ultrastruct. Res., 27, 216-229. 
WELLS, B. 1974. A convenient technique for the collection of ultra-thin serial sections. Micron., 5, 79-81.

whrTe, M. J. D. 1936. Chiasma localisation in Mecostethus grossus L. and Metrioptera brachyptera L. (Orthoptera). Z. Zellforsch., 24, 128-135.

wILson, E. B. 1925. The Cell in Development and Heredity, 3rd Edition. Macmillan, New York.

\section{ADDENDUM}

The localised development of SC's noted by Jones (1973) was occasionally seen in the insects used in the present study, but many individuals (including the insect used in the reconstructions presented here) completely lacked such asymmetrical structures. The relevance of these asymmetrical regions of the SC for the control of chiasma localisation is therefore very questionable, and they are probably best regarded as sporadic variants of SC structure, as noted in other organisms (e.g. Moens 1973; LaCour and Wells 1973).

MOENS, P. B. 1973. Quantitative electron microscopy of chromosome organisation at meiotic prophase. Cold Spring Harbór Symp. Quant. Biol., 38, 99-107.

LACOUR, L. F., AND WELLS, B. 1973. Deformed lateral elements in synaptinemal complexes of Phaedranassa viridifora. Chromosoma (Berl.), 41, 289-296. 